\title{
LA AUTONOMÍA UNIVERSITARIA, LA VIGENCIA DEL III CONGRESO UNIVERSITARIO Y UNA OBLIGADA REFERENCIA A RODRIGO FACIO
}

\author{
UNIVERSITY AUTONOMY, THE VALIDATION OF THE III UNIVERSITY \\ CONGRESS AND AN OBLIGATORY REFERENCE TO RODRIGO FACIO
}

\author{
Daniel Camacho Monge*
}

\section{RESUMEN}

El III Congreso Universitario es producto de un vigoroso movimiento de defensa de la autonomía universitaria, el cual se desarrolló en la Universidad de Costa Rica, al principio de la década de los años 70 del siglo XX, con participación de los estamentos: docente, estudiantil y administrativo, de todas las expresiones ideológicas y de las diversas generaciones presentes en la única Universidad de entonces. También se sostiene que sus resultados, plasmados en el Estatuto Orgánico, fueron de avanzada para el momento y continúan vigentes. En este contexto, es obligado el recuerdo del aporte de Rodrigo Facio, como un importante antecedente en la forja de la autonomía universitaria en Costa Rica.

PALABRAS CLAVE: RODRIGO FACIO * UNIVERSIDADES * AUTONOMÍA * GRUPOS DE INTERÉS

\section{ABSTRACT}

The III Congress University is a result of a vigorous movement to defend university autonomy. It was developed at the University of Costa Rica, at the beginning of the decade of the $70 \mathrm{~s}$ of the 20th Century, with the participation of strata: teachers, student and administrative, of all ideological expressions and the diverse generations present at the only University at that time. Also, it is supported that the results embodied in the Organic Statute, were advanced for the time and remain in force. In this context, it must be the memory of Rodrigo Facio's contribution as an important precedent in the forging of university autonomy in Costa Rica.

KEYWORDS: RODRIGO FACIO * UNIVERSITIES * AUTONOMY * INTEREST GROUPS

\footnotetext{
* Director de la Revista de Ciencias Sociales de la Universidad de Costa Rica.
} revista.cs@ucr.ac.cr 


\section{INTRODUCCIÓN}

Para comprender integralmente el proceso de transformación universitaria generado por el III Congreso Universitario de la Universidad de Costa Rica (UCR), celebrado entre 1973 y 1974, y su actual vigencia, conviene comenzar con la contextualización y reconstrucción histórico-social del III Congreso Universitario (Parte I) para continuar con el análisis de algunos de sus resultados fundamentales para la autonomía (Parte II) y concluir con algunas referencias a la vigencia de sus aportes (Parte III). El contenido de cada una de esas partes sería el siguiente:

PARTE I. Contexto histórico social del III Congreso Universitario

1. Costa Rica y el mundo al inicio de la década de los 70 del siglo Xx

2. La Universidad de Costa Rica en esa época

3. Los poderes fácticos de aquel momento y sus relaciones con la UCR

4. Breve recuento de algunos aspectos pertinentes de la historia concreta del III Congreso

PARTE II. Algunos resultados del III Congreso fundamentales para la autonomía

1. Los principios, propósitos y funciones

2. Las dos triadas. La triada humana: académicos/estudiantes/administrativos y la triada funcional: docencia/investigación/acción social

PARTE III. La vigencia del III Congreso en relación con la autonomía

PARTE IV. Rodrigo Facio, importante antecedente en la forja de la autonomía

PARTE I. CONTEXTO HISTÓRICO SOCIAL DEL III CONGRESO UNIVERSITARIO

El III Congreso Universitario (1973-1974) se desarrolló dentro de un dinámico ambiente de discusión política e ideológica que caracterizó la sociedad costarricense al final de los años 60 $y$ principios de los 70, en el cual la Universidad de Costa Rica — la única existente - jugó un importantísimo papel.

En la Universidad confluían las más variadas corrientes de pensamiento, pero las tendencias más críticas y cuestionadoras se expresaban en ella con mayor fuerza que en otros ámbitos, como era esperable, dada la libertad del medio universitario. Eso causó inquietud en círculos de poder, los cuales lograron que fuerzas $y$ actores influyentes dentro del Gobierno de turno, desarrollaran presiones, consistentes en anunciar condicionamientos para las transferencias presupuestarias a la Institución.

La respuesta de la comunidad universitaria - digo bien 'la comunidad' porque surgió de las bases docentes y estudiantiles- fue la convocatoria del III Congreso, con el objetivo de realizar un auto examen institucional y renovar el rumbo de la Universidad, antes de permitir injerencias externas.

Eso quiere decir que en la génesis misma del III Congreso, estaba fuertemente presente la defensa de la autonomía por parte, es cierto, de las autoridades de la Institución, pero más enérgicamente, por parte de un vigoroso movimiento de base que lo concibió, lo organizó, lo desarrolló y logró la profunda transformación de la concepción y la práctica universitarias en Costa Rica.

Hubo también otros factores que influyeron en la convocatoria, los cuales espero explicar o por lo menos, mencionar.

Para una cabal comprensión del III Congreso, es necesario ubicarlo en el complejo contexto histórico social en el cual se desarrolló, para lo cual se hace necesario describir qué pasaba en Costa Rica y en la Universidad durante esa época.

\section{COSTA RICA Y EL MUNDO AL INICIO DE LA DÉCADA DE LOS 70 DEL SIGLO XX}

El istmo centroamericano es vital en las relaciones planetarias en vista de su estratégica posición y Costa Rica absorbía con intensidad las contradicciones geopolíticas internacionales. La llamada Guerra Fría era intensa. Recuérdese que solo ocho años antes, en 1962, se produjo la crisis de los misiles cubanos, la cual 
puso al mundo al borde de la guerra nuclear $y$ que en ese contexto, las potencias occidentales exigían fuertemente la lealtad de los países latinoamericanos y muy especialmente de los centroamericanos, aunque eso significase que esas potencias sostuvieran regímenes dictatoriales.

También en esa época, eran palpables las consecuencias de la guerra civil costarricense de 1948, acaecida solo 20 años antes, durante los cuales se impuso, en Costa Rica, una especie de "pax romana" con predominio absoluto de las fuerzas - aunque diversas entre sí-que triunfaron en ese conflicto armado.

Pero al final de la década de los $60 y$ principios de los 70 , comienza a revigorizarse la resistencia, tanto de los trabajadores de las alejadas zonas bananeras como de los campesinos sin tierra, quienes empujados por el agotamiento de la frontera agrícola, realizaban ocupaciones de tierras acaparadas o estatales $y$ eran a menudo duramente reprimidos. Entre los trabajadores del Estado y sus instituciones se desarrollaron también organizaciones sindicales nuevas y cuestionadoras.

Por otro lado, la temprana Revolución Cubana despertó el interés de importantes sectores de la población, especialmente de las $y$ los jóvenes, por sus banderas de defensa de la soberanía nacional, la reivindicación de la historia y la cultura propias, y sus propósitos de justicia social.

Por esas razones, la "pax" surgida de la guerra de 1948 estaba llegando a su fin, pues fue cuestionada desde muy diversos sectores de la población y desde muy variados ámbitos políticos y sociales. Como era de esperarse, esa posición crítica también se manifestó en la Universidad.

\section{LA UNIVERSIDAD DE COSTA RICA EN ESA ÉPOCA}

Durante las largas dos décadas que hemos dado en llamar de la "pax romana", la Universidad, con Rodrigo Facio y Carlos Monge Alfaro a la cabeza, progresó considerablemente, se fortaleció, consolidó y creció, y supo reafirmar su autonomía. Todo ello sin cuestionar el régimen, sino por el contrario, contribuyendo a desarrollar el nuevo modelo social surgido a partir de 1948, al cual tanto bien le debe el país, sobre todo por las políticas sociales del merecidamente benemérito presidente José Figueres Ferrer y otros altos dirigentes, entre quienes destacan nuestros rectores Facio y Monge.

Pero los factores señalados anteriormente (apoyo imperial a las dictaduras en América Latina, fortalecimiento de las luchas obreras, campesinas y sindicales en Costa Rica, $y$ las banderas independentistas de la Revolución Cubana) hacen surgir en el país una fuerte resistencia, la cual como era de esperarse, tuvo no la única, pero sí una muy importante manifestación en el ámbito universitario.

Una de esas manifestaciones fue el surgimiento de grupos estudiantiles de tendencias contrarias a la liberacionista $y$ situados mucho más a la izquierda, los cuales pugnaron por, $y$ a menudo lograron, el control de la Federación de Estudiantes y de muchas otras organizaciones estudiantiles. Además, entre algunos profesores se compartía la práctica de involucrarse en los problemas nacionales $y$ apoyar los procesos que clamaban por más justicia social.

Muestra de la actitud crítica existente en el medio nacional, es la célebre y profunda resistencia a la concesión otorgada a la compañía Aluminiun Company of America (ALCOA) para la explotación de los yacimientos de bauxita. En los primeros meses de 1969, el movimiento contrario a esa concesión paralizó el país y logró detener la ejecución del contratoley del Estado con la compañía ALCOA, aún cuando ya estaba ratificado por la Asamblea Legislativa. En ese movimiento participaron activamente amplios sectores de la población costarricense, pero el movimiento estudiantil $y$ en general, los universitarios, incluidas casi todas las tendencias ideológicas y político partidistas, fue uno de sus ejes más importantes.

\section{LOS PODERES FÁCTICOS DE AQUEL MOMENTO Y SUS RELACIONES CON LA UCR}

Como se dijo, en los años 1970 y 1971, se cuestionó desde sectores del Gobierno, el presupuesto universitario y se insinuó la intención de revisar si la Institución ejecutaba políticas adecuadas al desarrollo del país y si invertía bien sus recursos.

Es posible que esa postura de algunos círculos gubernamentales obedeciera a una 
normal preocupación por el gasto público y a un deseo de asegurar una eficiente contribución de la Universidad al proyecto de desarrollo prevaleciente.

Pero también contribuyeron a esa posición gubernamental, los grupos del poder económico, que — como se mencionó antes- se sentían amenazados por los cuestionamientos de varios sectores de la población y propugnaban por un mayor control social del país, incluida la Universidad, sobre todo después de la resistencia al contrato-ley con la ALCOA y la fuerza que tomaron grupos de profesores $y$ estudiantes con posturas críticas. Esto es palpable si se revisa la gran prensa de la época.

En ese contexto histórico social se convoca al III Congreso para cuyo análisis se hace necesario reconstruir someramente su historia.

\section{ALGUNOS ASPECTOS PERTINENTES DE LA HISTORIA CONCRETA DEL III CONGRESO}

Ante la posibilidad de que el Gobierno se atreviera a inmiscuirse en los asuntos que la Constitución Política reserva a la Universidad, en reconocimiento de su autonomía, la reacción de la comunidad universitaria es digna de recordar con encomio. Todas las expresiones ideológicas, políticas, filosóficas y religiosas, incluidas aquellas muy afines al Gobierno, cerraron filas del lado universitario. Quienes conozcan a los universitarios de la época, podrán identificar a personas no solo afines, sino dirigentes importantes del partido gobernante, proponiendo $y$ defendiendo, dentro del III Congreso, tesis en pro de la autonomía.

Es cierto también que algunos importantes e influyentes personajes de la vida universitaria se opusieron enérgicamente a la idea misma de convocar a un Congreso y cuando no lo pudieron evitar, combatieron el carácter determinativo que intentábamos y logramos darle.

Desde unos años antes, el clima en la Universidad de Costa Rica era de intensa agitación intelectual. Se reunían grupos de profesores para discutir los temas universitarios $y$ nacionales, se desarrollaban debates por la prensa sobre diversos temas de la educación superior, se convocaba a mesas redondas sobre esos temas; en el medio estudiantil, la hegemonía de una tendencia terminó y la dirección de las organizaciones oficiales y no oficiales de los estudiantes fue disputada por grupos nuevos, con nuevas concepciones, los cuales emergieron con fuerza. Estaba fuertemente presente la preocupación por lo que sucedía en la realidad social del país y desde algunos grupos se reclamaba un mayor involucramiento de la Universidad en los problemas nacionales, cosa que algunos profesores $y$ estudiantes practicaban desde sus cátedras y sus organizaciones.

En ese ambiente, cayó el anuncio del interés de algún sector del Gobierno por supervisar a la Universidad. La reacción de los muchos y diversos grupos muestra una sorprendente coincidencia: la realización de un congreso que bloqueara esas intenciones y que produjera un examen autocrítico del pasado y una propuesta para el futuro, todo lo cual debía ser hecho por la comunidad universitaria, sin injerencia exterior. Es cierto que desde antes se sentía y se hablaba de la necesidad del Congreso, pero lo señalado fue un factor precipitante.

Todo ello muestra, como se dijo antes, que el III Congreso es producto de un vigoroso movimiento de defensa de la autonomía universitaria.

Fiel al origen participativo y colectivo, la convocatoria fue lo más amplia posible, sin restricciones para docentes interinos o que no pertenecieran a la entonces llamada Carrera Docente, con invitación a funcionarios administrativos y una cuota de estudiantes equivalente al 25\%, lo cual anticipaba la inclusión, a propuesta del III Congreso, de esa proporción en los órganos de Gobierno Universitario.

Las comisiones trabajaron largos periodos en 1971 y 1972, y sus propuestas fueron discutidas en Asambleas Plenarias celebradas en dos etapas, la primera, en octubre de 1971 y la segunda, en mayo de 1972. Las comisiones redactoras de las ponencias se integraron por grupos de profesores con participación de la cuota estudiantil y se procuró que en esas comisiones, estuvieran representadas las diversas tendencias de pensamiento, sin restricción alguna.

Las resoluciones definitivas del III Congreso fueron conocidas por la Asamblea Universitaria prevista en el Estatuto vigente, la 
cual celebró cinco sesiones para ello, durante el segundo semestre de 1972 y aprobó lo resuelto por el Congreso en forma casi intacta, con excepción de la estructura administrativa, la cual sí fue modificada.

Lo aprobado por esas Asambleas pasó a una comisión redactora cuyo informe fue al nuevo Consejo Universitario, integrado de acuerdo con la propuesta del Congreso, el cual aprobó definitivamente el nuevo estatuto. El trabajo de la Comisión Redactora del proyecto y las sesiones del nuevo Consejo Universitario consumieron todo el año 1973 y los primeros meses de 1974. El 15 de marzo de 1974, el Consejo Universitario aprobó en definitiva el nuevo Estatuto Orgánico, el cual fue publicado siete días después en $L a$ Gaceta, el 22 de marzo de 1974.

Por lo anterior, consideramos que el III Congreso comprende ese conjunto de instancias: comisiones, plenarias, asambleas universitarias, comisiones redactoras y sesiones del nuevo Consejo Universitario. Ese proceso parte en junio de 1971, con la Asamblea Universitaria que aprueba la convocatoria y culmina en marzo de 1974, con la publicación del nuevo Estatuto. Es un solo proceso rico en ideas y debates que recoge lo que podríamos llamar el espíritu del III Congreso.

La amplia participación en la convocatoria $y$ en sus trabajos dio a las propuestas del III Congreso un alto grado de legitimidad, lo cual explica la adopción casi literal de esas propuestas por parte de las instancias que las estudiaron posteriormente. Por ello, el nuevo Estatuto Orgánico, aprobado en marzo de 1974, recoge de manera casi literal las resoluciones del Congreso y de las asambleas que lo culminaron.

Como se anotó, la estructura administrativa surgida del Congreso fue modificada en la Asamblea Universitaria y ese hecho merece un poco de atención, ya que ese cambio no fue pacífico ni inocente.

A posteriori se puede constatar que obedecía a los planes gubernamentales en el campo de la expansión de la Educación Superior. Por ello, los profesores de Ciencias Económicas afines al Gobierno, con todo derecho y sin que eso empañe su buena fe, pues actuaban según sus convicciones, se empeñaron en introducir esos cambios.
La diferencia entre los dos modelos de Gobierno y Administración es la siguiente: en el III Congreso, el modelo de expansión de la Educación Superior consistía en la creación de nuevas sedes de la UCR, las cuales podrían, según su desarrollo, adquirir independencia con su propia Asamblea, su Consejo Universitario, su Rector, sus vicerrectores, sus escuelas $y$ facultades. Se creaba una Junta Universitaria Nacional, como ente superior, la cual estaría por encima de los Consejos Universitarios de las sedes. Se optó por este modelo para garantizar que la autonomía concedida por la Constitución Política a la UCR, cubriera a las nuevas instituciones estatales de Educación Superior que se crearan en el futuro.

Una muestra más de la opción por la Universidad frente a las pretensiones del Gobierno, por parte de profesores afines a él, pero auténticamente universitarios, fue la enérgica defensa que el Lic. Carlos José Gutiérrez, destacado dirigente del Partido de Gobierno, profesor y decano de la Facultad de Derecho, hizo de este modelo. Personalmente, lo recuerdo argumentando, en forma brillante, en el sentido de que la autonomía constitucional de la Universidad de Costa Rica, debería cubrir las otras instituciones de Educación Superior que se establecieran en el futuro. Se separaba así — con la honestidad que siempre lo caracterizó- de la que parecía ser la postura oficial del Gobierno que él apoyaba.

El modelo aprobado en definitiva es el vigente, del cual hay que decir que ha mostrado ser funcional y aceptable. Estableció Asamblea Universitaria, Consejo Universitario, Rectoría, vicerrectorías, sedes, facultades y escuelas, únicamente para la Universidad de Costa Rica. Sin decirlo expresamente, ese modelo implicaba que, si se constituían otras universidades estatales, se regularían por las leyes específicas que el Ejecutivo propondría a la Asamblea Legislativa, tal como se hizo posteriormente con la Universidad Estatal a Distancia (UNED) y el Instituto Tecnológico (TEC).

En un Gobierno posterior del mismo Partido, cuando se creó la Universidad Estatal a Distancia, Institución que ha desempeñado un papel muy positivo en la vida nacional, uno 
de los argumentos fue que una de las ventajas de la enseñanza a distancia estriba en que los alumnos no están reunidos y eso los aleja de la influencia de quienes se dedican a la organización de los estudiantes.

Sucedió lo mismo con la fundación del Instituto Tecnológico de Costa Rica. Esa magnífica institución se creó por medio de una ley restrictiva de la participación de los universitarios en la dirección institucional. Tuvo que ocurrir en 1980, aproximadamente ocho años después de su creación, una prolongada $y$ dura huelga de las y los estudiantes (con fuerte intervención policial y con estudiantes heridos, detenidos $y$ acusados judicialmente) para que la Asamblea Legislativa modificara la Ley, creara la Asamblea Universitaria, modificara la forma de elección del Consejo Universitario y estableciera la normativa y las condiciones para que el TEC adquiriera la capacidad de darse un gobierno propio. Una dura batalla ganada en pro de la autonomía universitaria, que a menudo se olvida.

De lo anterior, queda claro que la oposición entre los dos modelos de Gobierno Universitario, enfrentados en el III Congreso y sus subsiguientes asambleas universitarias, no eran una simple discusión de teoría y práctica de la administración, sino involucraban un importantísimo debate sobre la autonomía de las instituciones de Educación Superior que se crearían con posterioridad.

Falta mucho por comentar sobre el III Congreso, pero no es esta la ocasión. Escribí un análisis más amplio, el cual fue publicado en el número doble 49-50 del año 1992, de la Revista de Ciencias Sociales de nuestra Universidad.

Basten los antecedentes comentados para abordar otros aspectos de la relación entre algunos resultados del III Congreso y la autonomía universitaria, en la perspectiva de su actual vigencia.

\section{PARTE II. ALGUNOS RESULTADOS DEL III CONGRESO FUNDAMENTALES PARA LA AUTONOMÍA}

\section{LOS PRINCIPIOS, PROPÓSITOS Y FUNCIONES}

La definición de los principios, propósitos y funciones de la Universidad de Costa Rica, es un buen ejemplo de la confluencia de pareceres que existió como compromiso entre las fuerzas que se movieron durante el Congreso. Los autores de la ponencia que sirvió de base para la definición de los principios, propósitos y funciones de la UCR, fueron Guillermo Malavassi Vargas, Fernando Leal Arias, Jorge Enrique Guier e Hilda Chen Apuy. Quienes los conozcan, reconocerán la considerable diferencia entre sus respectivas opciones de pensamiento. Hay que decir con satisfacción que la propuesta no coincidió con alguna de sus posturas personales, porque fue clara, estricta y profundamente universitaria.

En cuanto a los principios, propósitos y funciones, el Estatuto de marzo de 1974, recoge literalmente la resolución correspondiente del Congreso y de este entresacamos los siguientes párrafos:

El propósito de la Universidad de Costa Rica es obtener las transformaciones que la sociedad necesita para el logro del bien común, mediante una política dirigida a la consecución de una verdadera justicia social, del desarrollo integral, de la libertad plena y de la total independencia de nuestro pueblo.

Para este propósito, la Universidad estimulará la formación de una conciencia creativa, crítica y objetiva en los miembros de la comunidad costarricense, que permita a los sectores populares participar eficazmente en los diversos procesos de la actividad nacional.

El propósito general y los objetivos inmediatos de la Universidad de Costa Rica demandan de ella la búsqueda constante, inagotable y libre, de la verdad, la eficacia y la belleza.

Son funciones de la Universidad de Costa Rica:

a) Contribuir al progreso de las ciencias, las artes, las humanidades y la técnica, 
reafirmando su interrelación y aplicándolas al conocimiento de la realidad costarricense.

b) Estudiar los problemas de la comunidad y participar en proyectos tendientes al pleno desarrollo de los recursos humanos, en función de un plan integral destinado a formar un régimen social justo, que elimine las causas que producen la ignorancia y la miseria, así como a evitar la indebida explotación de los recursos del país.

c) Contribuir a elevar el nivel cultural de la nación costarricense mediante la acción universitaria.

ch) Impulsar y desarrollar la enseñanza e investigación de alto nivel.

d) Formar un personal idóneo que se dedique a la enseñanza, las ciencias, las artes $y$ las letras, para que participe eficazmente en el desarrollo del sistema de educación costarricense.

e) Proporcionar a los estudiantes una cultura superior de orden general, como base y complemento de la formación especial o profesional.

f) Mantener la libertad de cátedra como principio de la enseñanza universitaria, que otorga a los miembros del claustro plena libertad para expresar sus convicciones filosóficas, religiosas y políticas.

g) Garantizar dentro del ámbito universitario el diálogo y la libre expresión de las ideas y opiniones, así como la coexistencia de las diferentes ideologías y corrientes del pensamiento filosófico, religioso $y$ político, sin otra limitación que el respeto mutuo.

h) Formar profesionales en todos los campos del saber capaces de transformar, provechosamente para el país, las fuerzas productivas de la sociedad costarricense $y$ de crear conciencia crítica en torno a los problemas de la dependencia y del subdesarrollo.

Hemos subrayado algunos párrafos singularmente significativos para mostrar que esa declaración de principios, propósitos y funciones constituye una reafirmación terminante de la autonomía, porque se centra en elevados fines, se perfila hacia grandes metas y se compromete con la construcción de una sociedad informada, reflexiva, crítica y con justicia social.

Nada más alejado de los fines utilitarios, inmediatistas $y$ mercantiles que entonces $y$ ahora, se quieren imponer desde fuera a la Universidad. He ahí otro de los rasgos que mantienen actuales $y$ vigentes los aportes de aquel lejano y célebre III Congreso.

\section{LAS DOS TRIADAS. LA TRIADA DE LOS RECURSOS HUMANOS: ACADÉMICOS/ESTUDIANTES/ ADMINISTRATIVOS Y LA TRIADA FUNCIONAL: DOCENCIA/INVESTIGACIÓN/ACCIÓN SOCIAL}

Aunque no quedó plasmado en forma terminante en algún acuerdo, uno de los productos más interesantes de los debates y del espíritu del III Congreso, es la integración del quehacer universitario en dos triadas que denominaré la de recursos humanos y la funcional. La triada de los recursos humanos es la integración en el trabajo concreto de los tres estamentos universitarios: académicos, estudiantes $y$ administrativos. La triada funcional es la integración de los quehaceres: docencia, investigación y acción social.

La triada de los recursos humanos implica el compromiso de realizar el trabajo universitario integrando los tres estamentos en cada acción. Eso sí, con los objetivos académicos como norte, punto que en la práctica a veces se olvida.

La triada funcional es la integración de la investigación, la docencia y la acción social en todo el quehacer universitario.

La combinación de ambas triadas implica que todo académico y todo estudiante investigue, que la investigación generalizada alimente la docencia y que los resultados se vuelquen hacia la sociedad.

En el Estatuto surgido del III Congreso es constante la reafirmación del compromiso de la Universidad con la sociedad para el logro del bien común, la consecución de una verdadera justicia social, la formación de una conciencia crítica en la población y la formación de profesionales capaces de crear esa conciencia 
crítica en pro de un régimen social justo, que elimine las causas que producen la ignorancia y la miseria, así como a evitar la indebida explotación de los recursos del país.

Esos altos propósitos requieren como mínimo dos requisitos: un régimen de autonomía porque hay en la sociedad fuerzas poderosas que actúan objetivamente en contra de ellos $y$ una estrecha coordinación entre los diversos quehaceres universitarios.

El Estatuto de 1974 (artículo 51, inciso C) encomendó al Vicerrector de Investigación "velar porque la investigación esté coordinada con la docencia y con los programas de acción social". Pero a lo largo de los documentos y debates se enfatizó mucho en el tema de esa deseable estrecha relación.

Esa relación entre docencia, investigación y acción social, aunque no en forma terminante, se recoge en el Estatuto vigente, cuando establece en su artículo 50, incisos ch y d, como función del Vicerrector de Docencia:

Velar porque la labor docente en la Universidad se lleve a cabo en forma eficiente y actualizada, en unidad de propósitos con la investigación, utilizando los sistemas más adecuados de enseñanza y evaluación.

Velar porque los cursos universitarios presten siempre especial cuidado a la vinculación de los conocimientos universales con la realidad nacional, desarrollando en el estudiante una capacidad de análisis y de crítica que le permita aplicar creativamente los conocimientos adquiridos.

Esto tiene que ver directamente con la autonomía, porque se aleja de la concepción tecnocrática de ciertos poderosos círculos, que propugnan una Universidad al servicio de las demandas coyunturales del mercado. El mercado, por supuesto, tiene mucha importancia, pero la Universidad concebida alrededor de esas dos triadas, más bien tendría efectos en la sociedad, entre los cuales no se descartaría la modificación del mercado.

\section{PARTE III. LA VIGENCIA DEL III CONGRESO EN} RELACIÓN CON LA AUTONOMÍA

Antes de desarrollar la tercera y última parte de esta exposición, debo admitir que he omitido caracterizar o definir el concepto mismo de autonomía. Hay varios trabajos interesantes desde el punto de vista jurídico, pero se habrá notado que el concepto utilizado en esta exposición va más allá de lo jurídico. Es un concepto histórico-social y por lo tanto, hermenéutico, lo cual implica un análisis más completo y complejo, que toma en cuenta tanto el entorno social como el devenir histórico y permite una mayor profundidad.

Dicho esto, podemos volver a la parte III de esta exposición.

Los principios, propósitos y funciones de la Universidad de Costa Rica, aprobados en el Estatuto de marzo de 1974, no pueden ser más actuales y vigentes, porque la sociedad costarricense y la Universidad no han podido, como se lo propuso el III Congreso: obtener las transformaciones que la sociedad necesita para el logro del bien común, mediante una política dirigida a la consecución de una verdadera justicia social, el desarrollo integral, la libertad plena y la total independencia de nuestro pueblo.

La Universidad de Costa Rica ha hecho una formidable contribución al país en ese sentido, pero queda mucho por hacer y quizás, sea una tarea que nunca acabe.

A pesar de varias modificaciones de los estatutos, la Universidad de Costa Rica ha tenido la sabiduría de conservar inalterados esos principios, funciones y propósitos en reconocimiento de su actualidad.

Como no es posible referirse a todos ellos, cabe concentrarse en uno de gran importancia $y$ es el del financiamiento externo de la investigación.

El artículo 51 del Estatuto de 1974, establece que corresponderá específicamente al Vicerrector de Investigación: "velar porque la investigación no esté subordinada a intereses extranjeros, ni a los que en alguna forma obstaculicen el desarrollo de Costa Rica”.

Esa frase también se ha conservado inalterada y llega hasta el Estatuto actual. 
Esto nos lleva a recordar que la autonomía no es solo frente a los poderes públicos, sino también en contra de ciertos intereses privados.

Desde ese punto de vista, hay que examinar de cerca la llamada cooperación nacional e internacional en el campo de los programas sociales, docentes y de investigación. Más frecuente de lo que parece a simple vista y aunque parezca paradójico, esa llamada cooperación, en vez de transferir ayuda hacia la Universidad o el país, logra poner nuestros recursos a su servicio, a causa de los condicionamientos.

Las instituciones internacionales de cooperación - gubernamentales o no gubernamentales - ofrecen sus recursos de acuerdo a sus propios planes. El ente local -estatal, universitario, gubernamental o no gubernamental- debe adecuarse a esos planes si quiere ser elegible. Si a esto agregamos la obligación de la institución que recibe, de aportar lo que se denomina la contrapartida, consistente en un porcentaje del costo del proyecto, resulta que por medio de esas contrapartidas, los supuestamente ayudados, ponen sus recursos humanos $y$ materiales al servicio del cumplimiento de los planes de la entidad supuestamente cooperante. A eso se agrega que la entidad cooperante tiene el poder $-y$ lo ejerce a menudo- de clausurar el proyecto si sus ejecutantes se separan de las condiciones impuestas. Hay numerosos casos concretos en los cuales esto ha sucedido. ¿En dónde queda entonces el ejercicio concreto de la autonomía para decidir sin ataduras las actividades universitarias?

Tengo menos conocimiento del mecanismo de la venta de servicios, pero considero que se debería hacer un análisis similar al respecto.

Es notable que, a pesar de varias modificaciones hechas al Estatuto en este largo periodo, los principios, propósitos y funciones elaborados por el III Congreso se han mantenido.

Sin embargo, hay un punto preocupante. He notado que cuando se organiza un nuevo congreso, flota en el aire el prurito de superar al Tercero y por ello, surgen iniciativas de reformas al Estatuto. Hay que aclarar que, aunque no les está vedado, los congresos no se instituyeron para reformar el Estatuto, sino para discutir temas relacionados con el rumbo de la Universidad. Entiendo que en este momento hay una comisión encargada de redactar un nuevo estatuto. Ignoro a qué obedece esa iniciativa y me cuesta encontrar una explicación. El riesgo de ese prurito reformista es la posibilidad de minar las definiciones básicas de los principios, propósitos y funciones aprobados en el III Congreso. No se trata de que sean normas pétreas, pero son el producto, como he tratado de mostrar, de un profundo movimiento de universitarios de todas las corrientes de pensamiento, quienes trabajaron por más de tres años, recogieron la historia de la Institución, debatieron a veces acaloradamente y lograron construir plural y colectivamente unas definiciones que han funcionado $y$ nos han ayudado a defender la autonomía por el no despreciable periodo de cuarenta años. No quiero aparecer como alguien que defiende ciegamente a un hijo predilecto. Pero siento el deber de, por lo menos, recomendar prudencia $y$ parsimonia en un asunto tan delicado.

Aprovecho para recordar que dentro de un año, el día 22 de marzo de 2014, fecha de su publicación en La Gaceta, se cumplirán cuarenta años de la entrada en vigencia del Estatuto Orgánico surgido del III Congreso. Expreso respetuosamente mi deseo de que la Universidad de Costa Rica conmemore esas efemérides y como Secretario General de ese cónclave, ofrezco modestamente mi colaboración.

\section{PARTE IV. RODRIGO FACIO, IMPORTANTE ANTECEDENTE EN LA FORJA DE LA AUTONOMÍA}

En sus Obras Completas, Rodrigo Facio advierte que:

... el día de la liquidación de la vida espiritual creadora de la Universidad, será el día en que la Universidad estuviera al servicio de un poder político, o de una confesión religiosa, o de una tendencia anti-religiosa mutiladora de la integridad de la vida interior, o de un sectarismo de la vida doctrinario, o de una discriminación racial, o de un privilegio económico, o de una distinción social. 
A lo largo de su trayectoria vital, Rodrigo Facio fue un forjador de una Universidad esencialmente autónoma. Así lo propuso y defendió, junto con un grupo de sus compañeros en la Asamblea Constituyente de 1949, en la cual consiguió aprobar un texto que sigue incólume hasta ahora $y$ que dice:

Artículo 84.- La Universidad de Costa Rica es una institución de cultura superior que goza de independencia para el desempeño de sus funciones $y$ de plena capacidad jurídica para adquirir derechos y contraer obligaciones, así como para darse su gobierno propio.

Después de los intentos por parte de sucesivos gobiernos para mantener bajo control a las nuevas universidades públicas, las luchas de los universitarios lograron que en junio de 1975, se agregara a ese artículo la frase "las demás instituciones de educación superior universitaria del Estado tendrán la misma independencia funcional e igual capacidad jurídica que la Universidad de Costa Rica”.

En el pensamiento y la acción de Rodrigo Facio también se encuentran antecedentes de algunas ideas centrales desarrolladas por el III Congreso Universitario, como la relación de la Universidad con la sociedad, el compromiso universitario en la búsqueda del bien común, la misión universitaria de ayudar a conseguir las transformaciones que la sociedad necesita para lograr la justicia social.

\section{CONCLUSIÓN}

Las fuerzas, nacionales e internacionales, partidarias de debilitar la autonomía, se reproducen y multiplican su poder; sienten que los principios, propósitos y funciones que nos hemos impuesto en ejercicio de nuestra autonomía, estorban los procesos de acumulación que les benefician.

Por eso, la autonomía universitaria debe ser defendida cada día y en cada acción. En momentos de crisis, las manifestaciones públicas, como concentraciones y marchas, son oportunas. Pero en ellas no se agota la defensa de la autonomía.

La verdadera defensa está en la legitimidad de esta Universidad de Costa Rica, que se encuentra - según lo muestran los estudios de organizaciones especializadasentre las mejores de América Latina y ocupa los primeros lugares de aceptación y confianza en la población costarricense. Ese es nuestro mejor bastión.

Para mantener ese prestigio protector de nuestra autonomía, debemos ser estrictos $y$ puntillosos en nuestro quehacer universitario. Sobrios y transparentes en el manejo de los recursos que la sociedad pone en nuestras manos con singular generosidad. Intolerantes con algún brote de corrupción que pueda aparecer. Volcados sobre el bienestar de los estudiantes quienes deben disfrutar de buenas condiciones para facilitarles su formación. Responsables y apasionados en nuestras tareas docentes. Cumplidores de los compromisos que hemos asumido con sectores de la población que participan en nuestros programas y con la sociedad en general. Creadores de iniciativas serias para respaldar procesos de solución de problemas nacionales.

Solo así defenderemos la autonomía contra tantos $y$ poderosos adversarios, haremos honor a los padres fundadores de la Universidad $y$ a quienes los siguieron y ya no están, pero dejaron en nuestras manos esta universidad excelente, libre y comprometida con las mejores causas en pro de la felicidad de nuestro pueblo. 